\title{
Ten-year Review of Patients with Resected Esophagogastric Junction Adenocarcinoma in the Philippine General Hospital
}

\author{
Shiela S. Macalindong, MD, ${ }^{1}$ Arturo S. Dela Peña, $\mathrm{MD}^{1}$ and Brian Buckley, $\mathrm{PhD}^{2}$ \\ ${ }^{1}$ Division of Surgical Oncology, Head $\mathcal{F}^{2}$ Neck, Breast, Skin $\mathcal{F}^{3}$ Soft Tissue, and Esophagogastric Surgery, Department of Surgery, \\ College of Medicine and Philippine General Hospital, University of the Philippines Manila \\ ${ }^{2}$ Department of Surgery, Philippine General Hospital, University of the Philippines Manila
}

\begin{abstract}
Objective. To describe the clinicopathologic profile, management, and outcomes of patients with esophagogastric junction (EGJ) adenocarcinoma in the local setting.

Methods. Data was obtained from patients who had curative surgery for EGJ adenocarcinoma from 2004-2013 in the Philippine General Hospital. We used student's T-tests, analysis of variance, chi-squared and Fisher's exact tests for comparisons and Cohen's kappa index for correlation. A $P$ value of less than or equal to 0.05 was considered significant.

Results. We included 88 patients (81.2\% male) with mean age of 55.2 years. Eight percent were clinical Siewert type I; $23.9 \%$ were type II; $15.9 \%$ were type III; and majority (52.3\%) were unknown type. Surgical approach and resection differed across the Siewert types $(\mathrm{P}<0.000)$. Thoracoabdominal approach $(72.7 \%)$ and distal esophagectomy with total gastrectomy (77.3\%) were the most common procedures. Many had at least pathologic T3 (80.6\%), N2 (54.5\%), and stage III (68.2\%) disease. Neoadjuvant and adjuvant chemotherapy was given in 1.2\% (1/82) and 48.6\% (18/37), respectively. In-hospital morbidity was 40\%; mortality was 4.5\%; 1 -year disease-free survival rate was $69.4 \%$; and overall survival rate was $76.5 \%$. Correlation was fair between preoperative and pathologic Siewert type $(P=0.003)$ and poor between clinical and pathologic stage $(P=0.115)$. Patients with recurrence had higher pathologic lymph nodes $(P=0.029)$ and more advanced stage $(P=0.022)$.
\end{abstract}

Conclusion. EGJ adenocarcinomas were locally advanced and had poor outcomes. Surgery should be individualized and multimodality approach considered.

Key Words: esophagogastric junction, gastroesophageal junction, adenocarcinoma

\section{INTRODUCTION}

Esophagogastric junction (EGJ) adenocarcinomas are a group of tumors whose center lies within $5 \mathrm{~cm}$ proximal and distal to the anatomic cardia, with involvement of the EGJ..$^{1-3}$ In recent years, its incidence has increased in Western and in some Asian populations. ${ }^{1,2,4-6}$ Due to its

Presented as poster at the International Digestive Disease Forum [IDDF] 2015, June 6-7, 2015, HongKong.

Corresponding author: Shiela S. Macalindong, MD

Division of Surgical Oncology, Head and Neck, Breast, Skin and Soft Tissue, and Esophagogastric Surgery

Department of Surgery

Philippine General Hospital

University of the Philippines Manila

Taft Avenue, Manila 1000, Philippines

Email: shielamacalindongmd@gmail.com borderline location between the esophagus and stomach, there is controversy as to whether EGJ adenocarcinoma should be classified and consequently managed as primarily an esophageal or gastric malignancy. Its staging follows the esophageal system of the American Joint Committee on Cancer (AJCC) 7 th edition. ${ }^{3}$ Using topographic anatomic criteria, EGJ adenocarcinomas have been classified according to Siewert type, thereby facilitating the choice of surgical approach. ${ }^{1,2}$ Type I tumors have their epicenter between 1 to 
$5 \mathrm{~cm}$ proximal to EGJ, type II tumors within $1 \mathrm{~cm}$ proximal and $2 \mathrm{~cm}$ distal to the EGJ, and type III tumors between 2 to $5 \mathrm{~cm}$ distal to the EGJ. ${ }^{1-3}$ Type I tumors are regarded as distal esophageal adenocarcinoma, postulated to arise from areas of Barrett's esophagus, and to involve the EGJ from above. ${ }^{1,2}$ Type II tumors are considered true carcinomas of the cardia arising from the EGJ itself. ${ }^{1,2}$ Type III tumors are thought to be subcardial gastric carcinoma that infiltrate the EGJ and distal esophagus from below. ${ }^{1,2}$

Surgery remains the only potentially curative modality. Various surgical approaches can be used, including transthoracic or transhiatal esophagectomy and total or proximal gastrectomy with distal esophagectomy via laparotomy or left thoracoabdominal approaches. ${ }^{7}$ Surgical approach does not appear to influence oncologic outcomes. ${ }^{7-13}$ Since less than $50 \%$ of patients with EGJ adenocarcinomas can undergo curative resections, ${ }^{7}$ there is much interest in using multimodality approach. Chemotherapy or chemoradiotherapy as neoadjuvant or adjuvant therapy has been shown to improve survival over surgery alone in EGJ adenocarcinoma; but majority of the trials included only a portion (10-20\%) of EGJ adenocarcinoma patients. ${ }^{14-20}$ Prognosis for the disease remains poor. . $^{1,2,5,7,21-23}$

In the Philippines, the burden of disease is unknown, as no distinction between EGJ and esophageal or gastric cancer is made in estimates. However, gastric and esophageal cancer were estimated to be the $7^{\text {th }}$ and $19^{\text {th }}$ leading new cancer sites and the $6^{\text {th }}$ and $17^{\text {th }}$ leading new causes of cancer death, respectively, in $2010 .{ }^{24}$ There is currently no local literature that describes our experience with EGJ adenocarcinoma.

This study sought to establish baseline information on patients with EGJ adenocarcinoma treated at the Philippine General Hospital (PGH), a tertiary referral center that has an extensive single-institution experience in the management of this condition, Specifically we aimed to describe clinicopathologic profile, diagnostic and therapeutic management, and important outcomes such as in-hospital morbidity and mortality, recurrence and survival at one year. Such information can be used to evaluate management approaches and outcomes in the light of international standards and inform the development of local guidelines.

\section{METHODS}

We included patients who were adults 18 years and older, with histologically proven adenocarcinoma of the EGJ and retrievable medical records, who underwent primary curative resection in PGH from January 1, 2004 to December 31, 2013. Patients with metastatic disease or those with concurrent or history of other malignancy were excluded.

Patients were identified using the Integrated Surgical Information Systems (ISIS) database and conference compilation of the General Surgery 1 Division. Medical records were retrieved from the PGH Medical Records
Division. For private patients, records were accessed from private clinics after obtaining permission from attending surgeons.

We collected demographic and clinical data such as age, gender, smoking and alcoholic beverage consumption history, body mass index, albumin, history of gastroesophageal reflux disease (GERD), co-morbidities, symptomatology, physical examination findings, diagnostic procedures, preoperative histopathology, clinical and pathologic staging and Siewert classification. We also collected data on the clinical course and surgical outcomes including surgical procedure, status of resection margins, lymphovascular space invasion, histologic grade, associated histopathologic findings (intestinal metaplasia, Helicobacter pylori, esophagitis, chronic gastritis), in-hospital morbidity and mortality, neoadjuvant or adjuvant treatments, recurrence, and 1-year disease-free and overall survival. Patients' data were anonymized by using codes.

Means (standard deviations, SD) were calculated for continuous variables and frequencies or proportions (count, \%) for categorical variables. Student's T tests or analysis of variance (ANOVA) were used to compare scale variables between patient groups. Chi-squared tests or Fisher's exact tests were used to compare distributions between groups of categorical variables. Correlation between clinical and pathologic staging and preoperative and pathologic Siewert type was determined using Cohen's kappa indices. All analyses were 2 -sided and the level of statistical significance was set at $P$ value $\leq 0.05$. SPSS 20.0.0 was used for all statistical analyses.

The study protocol was approved by the University of the Philippines Manila Research Ethics Board.

\section{RESULTS}

A total of 88 patients who underwent curative surgery for EGJ adenocarcinoma in PGH between January 1, 2004 and December 31, 2013, were included. Majority (61.4\%) of the surgeries were performed by attending surgeons and $38.6 \%$ by surgical trainees. Mean duration of follow-up was 447 days. Demographic and clinical characteristics are presented in Table 1. Full data were not available for all patients, so that the denominators for percentage estimates vary between analyses.

Of patients for whom relevant data were available, a large majority (99\%) underwent upper gastrointestinal endoscopy. Mean proximal extent of tumors was estimated at 37.1 (SD 3.2) $(n=46)$ from the incisors. One-third of 49 patients had complete obstruction endoscopically. Majority ( $82 \%$ or $45 / 55)$ had preoperative histopathologic diagnosis of adenocarcinoma while $3.6 \%(2 / 55)$ had squamous cell carcinoma. Ninety-one percent $(55 / 61)$ had computed tomographic evaluation, half $(26 / 55)$ of whom had both chest and abdominal imaging. Seventeen of 71 patients (23.9\%) had barium swallow. One patient underwent endoscopic 
Table 1. Demographic, risk, and clinical profile of patients with resected esophagogastric junction adenocarcinoma

\begin{tabular}{lc}
\hline Characteristic & $\%^{*}(\mathrm{n} / \mathrm{N})$ \\
\hline Age at diagnosis (years), mean (SD) & $55.2(12.9)$ \\
\hline Male & $81.2(72 / 88)$ \\
\hline Smoking history & $79.1(34 / 43)$ \\
\hline Smoking - pack-years, mean (SD) & $33.4(23.3)$ \\
\hline Alcohol consumption & $82.5(33 / 40)$ \\
\hline Body mass index (kg/m $\left.{ }^{2}\right)$, mean (SD) $(\mathrm{n})$ & $20.3(3.4)(\mathrm{n}=40)$ \\
\hline Albumin (g/L), mean (SD) (n) & $33.0(5.7)(\mathrm{n}=49)$ \\
\hline History of GERD & $1.6 \%(1 / 61)$ \\
\hline Comorbidities & \\
Any & $57.4(35 / 61)$ \\
Hypertension & $32.8(20 / 61)$ \\
Pulmonary tuberculosis & $14.8(9 / 61)$ \\
Diabetes mellitus & $11.5(7 / 61)$ \\
Chronic obstructive pulmonary disease & $11.5(7 / 61)$ \\
Coronary artery disease & $4.9(3 / 61)$ \\
Others & $6.6(4 / 61)$ \\
\hline FEV1 & \\
Mean (\% predicted) & $86.3(18.1)$ \\
$\geq 80 \%$ predicted & $75.0(12 / 16)$ \\
$\geq 50-80 \%$ predicted & $18.8(3 / 16)$ \\
$\geq 30-50 \%$ predicted & $6.3(1 / 16)$ \\
<30\% & 0 \\
\hline Presenting symptom & \\
Dysphagia & $64.5(49 / 76)$ \\
Abdominal pain & $18.4(14 / 76)$ \\
Vomiting & $3.9(3 / 76)$ \\
Early satiety & $2.6(2 / 76)$ \\
Hematemesis & $2.6(2 / 76)$ \\
Others & $68.0(51 / 75)$ \\
\hline Associated symptoms & $48.0(36 / 75)$ \\
Weight loss & $26.7(20 / 75)$ \\
Nausea/vomiting & $21.3(16 / 75)$ \\
Abdominal pain & $26.7(20 / 75)$ \\
Early satiety & $17.3(13 / 75)$ \\
Melena/hematemesis & $48.0(36 / 75)$ \\
Anorexia & \\
Others & \\
\hline
\end{tabular}

\begin{tabular}{|c|c|}
\hline Characteristic & $\% *(n / N)$ \\
\hline $\begin{array}{l}\text { Physical examination findings } \\
\text { Normal } \\
\text { Pallor } \\
\text { Abdominal tenderness } \\
\text { Abdominal mass } \\
\text { Blood on digital rectal examination } \\
\text { Others }\end{array}$ & $\begin{array}{c}71.0(54 / 75) \\
12.0(9 / 75) \\
9.3(7 / 75) \\
4.0(3 / 75) \\
2.7(2 / 75) \\
2.7(2 / 75)\end{array}$ \\
\hline $\begin{array}{l}\text { Clinical Siewert type } \\
\text { Type I } \\
\text { Type II } \\
\text { Type III } \\
\text { Unknown }\end{array}$ & $\begin{array}{c}8.0(7 / 88) \\
23.9(21 / 88) \\
15.9(14 / 88) \\
52.3(46 / 88)\end{array}$ \\
\hline $\begin{array}{l}\text { Clinical stage } \\
\text { I } \\
\text { IIA } \\
\text { IIB } \\
\text { IIIA } \\
\text { IIIB } \\
\text { IIIC } \\
\text { IV } \\
\text { Unknown }\end{array}$ & $\begin{array}{c}1.1(1 / 88) \\
2.3(2 / 88) \\
28.4(25 / 88) \\
12.5(11 / 88) \\
0(0 / 88) \\
(1 / 88) \\
(1 / 88) \\
53.4(47 / 88)\end{array}$ \\
\hline $\begin{array}{l}\text { Clinical tumor status (T) } \\
\text { T1 } \\
\text { T2 } \\
\text { T3 } \\
\text { T4 } \\
\text { Unknown }\end{array}$ & $\begin{array}{c}0 \\
4.5(4 / 88) \\
46.6(41 / 88) \\
0 \\
48.9(43 / 88)\end{array}$ \\
\hline $\begin{array}{l}\text { Clinical nodal status (N) } \\
\text { N0 } \\
\text { N1 } \\
\text { N2 } \\
\text { N3 } \\
\text { Unknown }\end{array}$ & $\begin{array}{c}30.7(27 / 88) \\
13.6(12 / 88) \\
0 \\
2.3(2 / 88) \\
53.4(47 / 88)\end{array}$ \\
\hline
\end{tabular}

SD, Standard deviation; GERD, Gastroesophageal reflux disease; FEV, Forced expiratory volume; *percentage of patients for whom data were available

ultrasound, which was uninformative due to complete tumor obstruction. None underwent diagnostic laparoscopy.

Data on surgical approach and resection are presented in Table 2. Most of the cases were approached through a left thoracoabdominal incision, and resection frequently involved distal esophagectomy and total gastrectomy. Surgical approach and resection differed significantly according to clinical Siewert type.

The different types of reconstruction used after specific kinds of resection were: Roux-en Y esophagojejunostomy (distal esophagectomy and total gastrectomy), thoracic esophagogastrostomy (distal esophagectomy and proximal gastrectomy), cervical esophagogastrostomy (total thoracic esophagectomy and proximal gastrectomy), end cervical esophagostomy (total thoracic esophagectomy and total gastrectomy). En-bloc resection of additional organs was done in $31.8 \%$ of patients (28/88); the spleen removed in $96.4 \%$, the pancreas in $28.6 \%$ and the left adrenal gland in $1.1 \%$.
Data on histopathologic and clinical outcomes for all patients and for each pathologic Siewert type are shown in Table 3. The majority of patients had at least pathologic T3, N2, and stage III disease with clear proximal and distal resection margins. No significant differences in histopathologic characteristics, recurrence rates, and oneyear disease-free survival were found among the different Siewert types. However, one-year overall survival was significantly different; best survival was observed with pathologic Siewert type II. Data on circumferential/radial resection margin, lymphovascular invasion, and associated histopathologic findings was infrequently reported. Oneyear disease-free (69.4\%) and overall survival (76.5\%) were low but there was only a small proportion of patients with sufficient follow-up.

Around 58\% (21/36) of patients with long-term followup developed recurrence; average time to recurrence was 384 days $(\mathrm{SD}=212)$. Locoregional recurrence was most common 
Table 2. Surgical approach and resection by clinical Siewert type

\begin{tabular}{|c|c|c|c|c|}
\hline & \multicolumn{4}{|c|}{$\%(n / N)$} \\
\hline \multicolumn{5}{|l|}{ Surgical approach } \\
\hline Left thoracoabdominal & \multicolumn{4}{|c|}{$72.7(64 / 88)$} \\
\hline Transhiatal & \multicolumn{4}{|c|}{$4.5(4 / 88)$} \\
\hline Laparotomy + cervicotomy & \multicolumn{4}{|c|}{$10.2(9 / 88)$} \\
\hline Thoracotomy + laparotomy & \multicolumn{4}{|c|}{$1.1(1 / 88)$} \\
\hline Unspecified & \multicolumn{4}{|c|}{$11.4(10 / 88)$} \\
\hline \multicolumn{5}{|l|}{ Surgical resection } \\
\hline Distal esophagectomy + Total gastrectomy & \multicolumn{4}{|c|}{$77.3(68 / 88)$} \\
\hline Distal esophagectomy + Proximal gastrectomy & \multicolumn{4}{|c|}{$8.0(7 / 88)$} \\
\hline Total thoracic esophagectomy + Proximal gastrectomy & \multicolumn{4}{|c|}{$12.5(11 / 88)$} \\
\hline Total thoracic esophagectomy + Total gastrectomy & \multicolumn{4}{|c|}{$1.1(1 / 88)$} \\
\hline \multirow[t]{3}{*}{ Thoracic esophagectomy } & \multicolumn{4}{|c|}{$1.1(1 / 88)$} \\
\hline & \multicolumn{3}{|c|}{ Clinical Siewert Type } & \multirow{2}{*}{$P$ value } \\
\hline & I & II & III & \\
\hline Surgical approach & & & & $0.00^{*}$ \\
\hline Left thoracoabdominal & $42.9(3 / 7)$ & $90.0(18 / 20)$ & $100(13 / 13)$ & \\
\hline Transhiatal & 0 & $10.0(2 / 20)$ & 0 & \\
\hline Laparotomy + cervicotomy & $57.1(4 / 7)$ & 0 & 0 & \\
\hline Thoracotomy + laparotomy & 0 & 0 & 0 & \\
\hline Surgical resection & & & & $0.00^{*}$ \\
\hline Distal esophagectomy + total gastrectomy & $14.3(1 / 7)$ & $81(17 / 21)$ & $92.9(13 / 14)$ & \\
\hline Distal esophagectomy + proximal gastrectomy & 0 & $14.3(3 / 21)$ & $7.1(1 / 14)$ & \\
\hline Total thoracic esophagectomy + proximal gastrectomy & $71.4(5 / 7)$ & $4.8(1 / 21)$ & 0 & \\
\hline Total thoracic esophagectomy + total gastrectomy & $14.3(1 / 7)$ & 0 & 0 & \\
\hline
\end{tabular}

${ }^{*}$ Chi-squared test; Bold font, statistically significant $P$ value

Table 3. Clinicopathologic outcomes of resected esophagogastric junction adenocarcinoma overall and by pathologic Siewert type

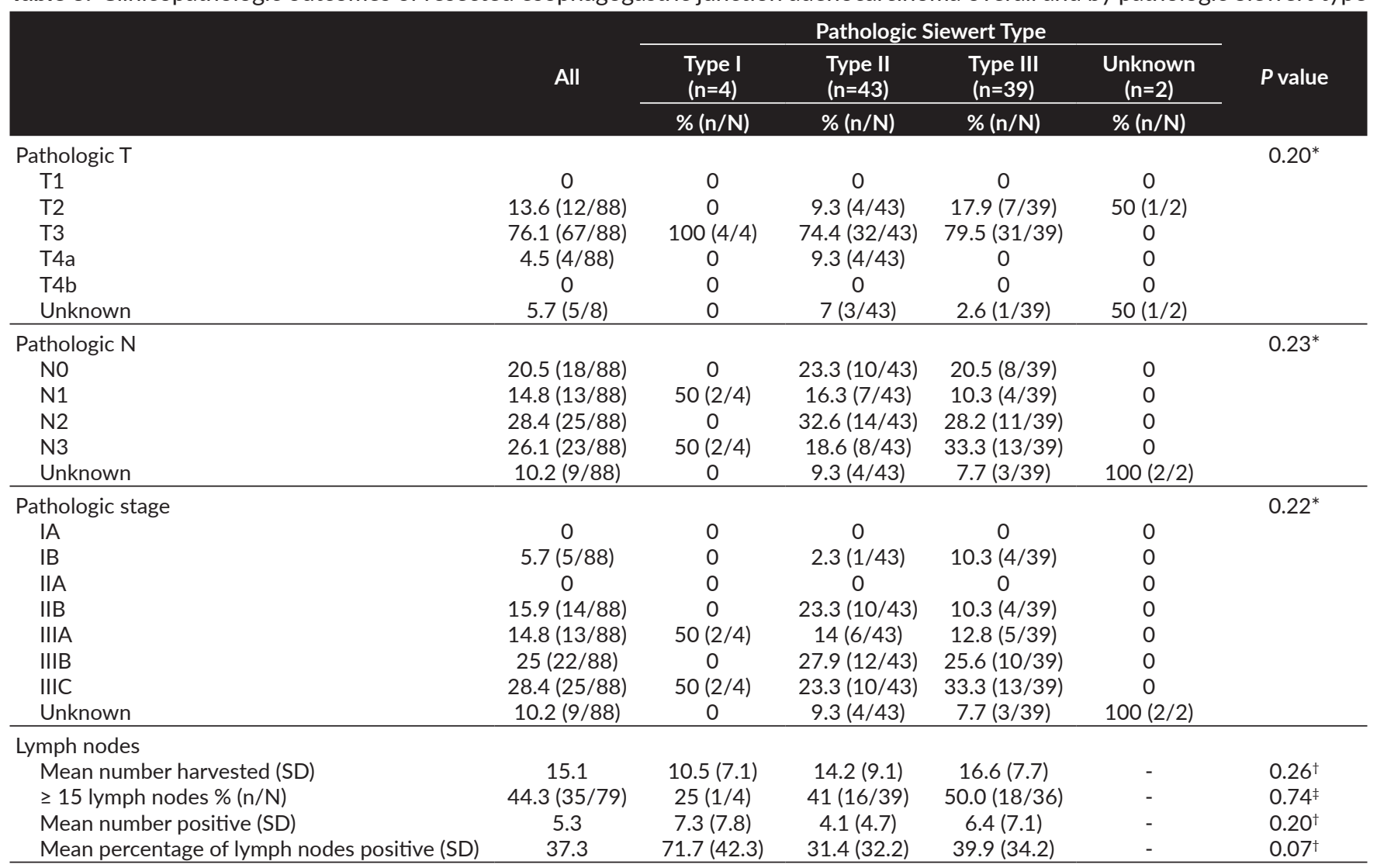


Table 3. Clinicopathologic outcomes of resected esophagogastric junction adenocarcinoma overall and by pathologic Siewert type (continued)

\begin{tabular}{|c|c|c|c|c|c|c|}
\hline \multirow{3}{*}{$x^{2}$} & \multirow{3}{*}{ All } & \multicolumn{4}{|c|}{ Pathologic Siewert Type } & \multirow{3}{*}{$P$ value } \\
\hline & & $\begin{array}{l}\text { Type I } \\
(\mathrm{n}=4)\end{array}$ & $\begin{array}{l}\text { Type II } \\
(\mathrm{n}=43)\end{array}$ & $\begin{array}{c}\text { Type III } \\
(\mathrm{n}=39)\end{array}$ & $\begin{array}{c}\text { Unknown } \\
(\mathrm{n}=2)\end{array}$ & \\
\hline & & $\%(n / N)$ & $\%(\mathrm{n} / \mathrm{N})$ & $\%(n / N)$ & $\%(n / N)$ & \\
\hline $\begin{array}{l}\text { Proximal margin } \\
\text { Positive } \\
\text { Negative } \\
\text { Unknown } \\
\text { Length }(\mathrm{cm}) \\
\end{array}$ & $\begin{array}{c}5.7(5 / 88) \\
84.1(74 / 88) \\
10.2(9 / 88) \\
2.4 \\
\end{array}$ & $\begin{array}{c}0 \\
100(4 / 4) \\
0 \\
4.5 \\
\end{array}$ & $\begin{array}{c}9.3(4 / 43) \\
83.7(36 / 43) \\
7(3 / 43) \\
2 \\
\end{array}$ & $\begin{array}{c}2.6(1 / 39) \\
87.2(34 / 39) \\
10.3(4 / 39) \\
3.1 \\
\end{array}$ & $\begin{array}{c}0 \\
0 \\
100(2 / 2) \\
- \\
\end{array}$ & $0.39 *$ \\
\hline $\begin{array}{l}\text { Distal margin } \\
\text { Positive } \\
\text { Negative } \\
\text { Unknown } \\
\text { Length }(\mathrm{cm}) \\
\end{array}$ & $\begin{array}{c}2.3(2 / 88) \\
87.5(77 / 88) \\
10.2(9 / 88) \\
7.9 \\
\end{array}$ & $\begin{array}{c}0 \\
100(4 / 4) \\
0 \\
5.3 \\
\end{array}$ & $\begin{array}{c}2.3(1 / 43) \\
90.7(39 / 43) \\
7.0(3 / 43) \\
7 \\
\end{array}$ & $\begin{array}{c}2.6(1 / 39) \\
87.2(34 / 39) \\
10.3(4 / 39) \\
9.7 \\
\end{array}$ & $\begin{array}{c}0 \\
0 \\
100(2 / 2) \\
- \\
\end{array}$ & $0.94^{*}$ \\
\hline $\begin{array}{l}\text { Circumferential margin } \\
\text { Positive } \\
\text { Negative } \\
\text { Close } \\
\text { Unknown } \\
\text { Width } \\
\end{array}$ & $\begin{array}{c}4.5(4 / 88) \\
5.7(5 / 88) \\
2.3(2 / 88) \\
87.5(77 / 88) \\
0.04 \\
\end{array}$ & $\begin{array}{c}0 \\
25.0(1 / 4) \\
0 \\
75.0(3 / 4) \\
- \\
\end{array}$ & $\begin{array}{c}7.0(3 / 43) \\
7.0(3 / 43) \\
4.7(2 / 43) \\
81.4(35 / 43) \\
0.04 \\
\end{array}$ & $\begin{array}{c}2.6(1 / 39) \\
2.6(1 / 39) \\
0 \\
94.9(37 / 39) \\
0 \\
\end{array}$ & $\begin{array}{c}0 \\
0 \\
0 \\
100(2 / 2) \\
- \\
\end{array}$ & $0.74^{*}$ \\
\hline $\begin{array}{l}\text { Lymphovascular invasion } \\
\text { Positive } \\
\text { Negative } \\
\text { Unknown } \\
\end{array}$ & $\begin{array}{c}11.4(10 / 88) \\
3.4(3 / 88) \\
85.2(75 / 88) \\
\end{array}$ & $\begin{array}{c}0 \\
0 \\
100(4 / 4) \\
\end{array}$ & $\begin{array}{c}14(6 / 43) \\
7.0(3 / 43) \\
79.1(34 / 43) \\
\end{array}$ & $\begin{array}{c}10.3(4 / 39) \\
0 \\
89.7(35 / 39) \\
\end{array}$ & $\begin{array}{c}0 \\
0 \\
100(2 / 2) \\
\end{array}$ & $0.50^{\ddagger}$ \\
\hline $\begin{array}{l}\text { Histologic grade } \\
\text { G1 } \\
\text { G2 } \\
\text { G3 } \\
\text { Unknown } \\
\end{array}$ & $\begin{array}{c}26.1(23 / 88) \\
27.3(24 / 88) \\
36.4(32 / 88) \\
10.2(9 / 88) \\
\end{array}$ & $\begin{array}{c}75.0(3 / 4) \\
0 \\
25.0(1 / 4) \\
0 \\
\end{array}$ & $\begin{array}{c}30.2(13 / 43) \\
30.2(13 / 43) \\
32.6(14 / 43) \\
7.0(3 / 43) \\
\end{array}$ & $\begin{array}{c}17.9(7 / 39) \\
28.2(11 / 39) \\
41.0(16 / 39) \\
12.8(5 / 39) \\
\end{array}$ & $\begin{array}{c}0 \\
0 \\
50.0(1 / 2) \\
50.0(1 / 2)\end{array}$ & $0.20^{*}$ \\
\hline $\begin{array}{l}\text { Associated histopathologic } \mathrm{f} \\
\text { H. pylori } \\
\text { Intestinal metaplasia }\end{array}$ & $\begin{array}{l}1.2(1 / 88) \\
2.4(2 / 88) \\
\end{array}$ & $\begin{array}{l}0 \\
0\end{array}$ & $\begin{array}{c}0 \\
2.3(1 / 43) \\
\end{array}$ & $\begin{array}{l}2.6(1 / 39) \\
2.6(1 / 39) \\
\end{array}$ & $\begin{array}{l}0 \\
0 \\
\end{array}$ & \\
\hline $\begin{array}{l}\text { Recurrence rate } \\
\text { Locoregional recurrence } \\
\text { Distant metastases }\end{array}$ & $\begin{array}{c}58.3(21 / 36) \\
44.4(16 / 36) \\
22.2(8 / 36) \\
\end{array}$ & $\begin{array}{c}50.0(1 / 2) \\
50.0(1 / 2) \\
0 \\
\end{array}$ & $\begin{array}{l}47.4(9 / 19) \\
47.4(9 / 19) \\
15.8(3 / 19) \\
\end{array}$ & $\begin{array}{c}73.3(11 / 15) \\
40(6 / 15) \\
33.3(5 / 15) \\
\end{array}$ & - & $0.30^{*}$ \\
\hline 1-year disease-free survival & $69.4(25 / 36)$ & $50(1 / 2)$ & $84.2(16 / 19)$ & $53.3(8 / 15)$ & $0(0 / 1)$ & $0.13^{*}$ \\
\hline 1-year overall survival & $76.5(26 / 34)$ & $33.3(1 / 3)$ & $94.4(17 / 18)$ & $66.7(8 / 12)$ & $0(0 / 1)$ & $0.03^{*}$ \\
\hline
\end{tabular}

${ }^{*}$ Chi-squared test

${ }^{\dagger}$ One-way analysis of variance (ANOVA)

¥Fisher's exact test

$(44.4 \%, 16 / 36)$ followed by distant metastases $(13.9 \%, 5 / 36)$ and concurrent locoregional and distant recurrence $(8.3 \%$, 3/36). Peritoneal recurrence was the most common site (19.4\%) followed by liver (13.9\%), regional lymph nodes (11.1\%), lungs (5.6\%) and anastomosis, tumor bed, and brain $(2.8 \%$ each).

Data relating to chemotherapy and radiotherapy was not available for all cases. For patients with data, around half (18/37) received adjuvant chemotherapy for an average of 4.1 cycles. Of the 17 patients whose chemotherapy regimens were known, 29\% each used epirubicin-cisplatin-fluorouracil and doxorubicin-cisplatin-fluorouracil combinations and $11.8 \%$ used single-agent capecitabine. Only $1.2 \%(1 / 82)$ of patients received neoadjuvant chemotherapy with one cycle of doxorubicin-cisplatin-fluorouracil. Two patients received adjuvant chemoradiotherapy (1 fluorouracil/leucovorin-based, 1 capecitabine-based).
In-hospital morbidity and mortality rates were $40 \%$ and $4.5 \%$, respectively. Pneumonia was the most common complication $(23.1 \%, 15 / 64)$ followed by superficial surgical site infection $(12.3 \%, 8 / 64)$, anastomotic leak [2 cervical esohagogastrostomy, 2 esophagojejunostomy, 1 tube jejunostomy] $(9.2 \%, 6 / 64)$, empyema thoracis $(4.6 \%, 3 / 64)$ and myocardial infarction $(3.1 \%, 2 / 64)$. No statistically significant difference in morbidity rates $(\mathrm{P}=1.00)$ and type $(\mathrm{P}=1.00)$ for pneumonia, superficial surgical site infection, and anastomotic leak was observed between thoracoabdominal and combined transhiatal/laparotomy and cervicotomy approaches. The four in-hospital deaths were due to sepsis from anastomotic leak, health-care associated pneumonia, myocardial infarction, hypovolemic shock due to mediastinal bleeding, and one unspecified cause. The correlation between preoperative Siewert type, obtained from endoscopic and CT scan information, and pathologic Siewert type was fair 
Table 4. Lymph node harvest, margin status, and clinical outcomes for resected esophagogastric junction adenocarcinoma by surgical approach and resection

\begin{tabular}{|c|c|c|c|c|c|c|c|c|}
\hline & \multicolumn{2}{|c|}{ Surgical Approach } & \multirow{3}{*}{$P$ value } & \multicolumn{4}{|c|}{ Surgical Resection } & \multirow{3}{*}{$P$ value } \\
\hline & $\begin{array}{c}\text { Transthoracic } \\
(n=64)\end{array}$ & $\begin{array}{c}\text { Non- } \\
\text { transthoracic } \\
(n=13)\end{array}$ & & $\begin{array}{c}\text { DE+TG } \\
(=\mathrm{DT}) \\
(\mathrm{n}=69)\end{array}$ & $\begin{array}{c}\text { TE } \pm P G \\
(=T P) \\
(n=12)\end{array}$ & $\begin{array}{c}\mathrm{DE}+\mathrm{PG} \\
(=\mathrm{DP}) \\
(\mathrm{n}=6)\end{array}$ & $\begin{array}{c}\mathrm{TE}+\mathrm{TG}(=\mathrm{TT}) \\
(\mathrm{n}=1)\end{array}$ & \\
\hline & $\%(n / N)$ & $\%(n / N)$ & & $\%(n / N)$ & $\%(n / N)$ & $\%(n / N)$ & $\%(n / N)$ & \\
\hline $\begin{array}{l}\text { No. of lymph nodes harvested } \\
\text { Mean } \\
\geq 15 \text { lymph nodes }\end{array}$ & $\begin{array}{c}15.0(n=11) \\
38.6(22 / 57)\end{array}$ & $\begin{array}{c}15.2(n=11) \\
25(1 / 4)\end{array}$ & $\begin{array}{l}0.94^{*} \\
0.16^{\dagger}\end{array}$ & $\begin{array}{c}15.5(n=64) \\
45.3(29 / 64)\end{array}$ & $\begin{array}{l}14.8(n=9) \\
44.4(4 / 9)\end{array}$ & $\begin{array}{c}10.4(n=5) \\
20(1 / 5)\end{array}$ & $\begin{array}{c}18(n=1) \\
100(1 / 1)\end{array}$ & $\begin{array}{l}0.62^{*} \\
0.54^{\dagger}\end{array}$ \\
\hline \% lymph nodes positive (mean) & $34.7(n=58)$ & $41.7(n=11)$ & $0.54^{*}$ & $34.5(n=64)$ & $47.4(n=9)$ & $42.9(n=5)$ & $94.4(n=1)$ & $0.25^{*}$ \\
\hline $\begin{array}{l}\text { Proximal margin status } \\
\text { Positive } \\
\text { Negative } \\
\text { Unknown } \\
\text { Margin length (mean, cm) }\end{array}$ & $\begin{array}{c}6.3(4 / 64) \\
82.8(53 / 64) \\
10.9(7 / 64) \\
2.3(n=35) \\
\end{array}$ & $\begin{array}{c}7.7(1 / 13) \\
84.7(11 / 13) \\
7.7(1 / 13) \\
3.1(n=9) \\
\end{array}$ & $0.87^{\ddagger}$ & $\begin{array}{c}5.8(4 / 69) \\
85.5(59 / 69) \\
8.7(6 / 69) \\
1.7 \\
\end{array}$ & $\begin{array}{c}8.3(1 / 12) \\
75(9 / 12) \\
16.7(2 / 12) \\
4.6 \\
\end{array}$ & $\begin{array}{c}0 \\
83.3(5 / 6) \\
16.7(1 / 6) \\
3.8 \\
\end{array}$ & $\begin{array}{c}0 \\
100(1 / 1) \\
0 \\
- \\
\end{array}$ & $0.02^{*}$ \\
\hline $\begin{array}{l}\text { Distal margin status } \\
\text { Positive } \\
\text { Negative } \\
\text { Unknown } \\
\text { Margin length (mean, cm) }\end{array}$ & $\begin{array}{c}0 \\
89.1(57 / 64) \\
10.9(7 / 64) \\
9.4(n=21) \\
\end{array}$ & $\begin{array}{c}15.4(2 / 13) \\
76.9(10 / 13) \\
7.7(1 / 13) \\
2.3(n=6) \\
\end{array}$ & $0.002^{\ddagger}$ & $\begin{array}{c}0 \\
91.3(63 / 69) \\
8.7(6 / 69) \\
10.5 \\
\end{array}$ & $\begin{array}{c}18.2(2 / 11) \\
72.7(8 / 11) \\
9.1(1 / 11) \\
2.7 \\
\end{array}$ & $\begin{array}{c}0 \\
83.3(5 / 6) \\
16.7(1 / 6) \\
5.0 \\
\end{array}$ & $\begin{array}{c}0 \\
100(1 / 1) \\
0 \\
- \\
\end{array}$ & $0.003^{\ddagger}$ \\
\hline In-hospital morbidity rate & $41.2(21 / 51)$ & $40.0(4 / 10)$ & $0.95^{\ddagger}$ & $42.6(20 / 47)$ & $30.0(3 / 10)$ & $33.3(2 / 6)$ & $100(1 / 1)$ & $0.55^{\ddagger}$ \\
\hline In-hospital mortality rate & $4.7(3 / 64)$ & $7.7(1 / 13)$ & $0.66^{\ddagger}$ & $4.3(3 / 69)$ & $0(0 / 12)$ & $0(0 / 6)$ & $100(1 / 1)$ & $0.00^{\ddagger}$ \\
\hline Recurrence rate & $45.8(11 / 32)$ & $75.0(6 / 8)$ & $0.15^{\ddagger}$ & $57.7(15 / 26)$ & $57.1(4 / 7)$ & $66.7(2 / 3)$ & - & $0.95^{\ddagger}$ \\
\hline 1-year disease-free survival & $75.0(18 / 24)$ & $62.5(5 / 8)$ & $0.50^{\ddagger}$ & $65.4(17 / 26)$ & $85.7(6 / 7)$ & $66.7(2 / 3)$ & - & $0.58^{\ddagger}$ \\
\hline 1-year overall survival rate & $79.2(19 / 24)$ & $71.4(5 / 7)$ & $0.67^{\ddagger}$ & $75.0(18 / 24)$ & $100(6 / 6)$ & $66.7(2 / 3)$ & $0(0 / 1)$ & $0.15^{\ddagger}$ \\
\hline
\end{tabular}

DE, Distal esophagectomy; TG, Total gastrectomy; TE, Total thoracic esophagectomy; PG, Proximal gastrectomy

${ }^{*}$ One way analysis of variance (ANOVA)

tFisher's exact test

${ }^{\ddagger}$ Chi-squared test

Bold font, statistically significant $P$ value

Notes:

Transthoracic approach $=$ Thoracoabdominal or Thoracotomy + Laparotomy

Non-transthoracic approach $=$ Transhiatal or Laparotomy + Cervicotomy

(Cohen's kappa, 0.342, $\mathrm{P}=0.003$ ), but was poor between clinical and pathologic staging (Cohen's kappa, 0.090, $\mathrm{P}=0.115)$.

Comparison of lymph node harvest, margins, and clinical outcomes for the different surgical approaches and resections are provided in Table 4. Only distal margin status $(\mathrm{P}=0.002)$ and length $(\mathrm{P}=0.001)$ were significantly different between transthoracic and non-transthoracic surgical approaches. Across different surgical resections, there were statistically significant differences in proximal margin length $(\mathrm{P}=0.017)$, distal margin status $(\mathrm{P}=0.003)$ and length $(\mathrm{P}=0.00)$, and in-hospital mortality rate $(\mathrm{P}=0.00)$. Except for the transthoracic approach and distal esophagectomy + total gastrectomy, other surgical approaches and resections had very limited observations.

The clinicopathologic and treatment factors in patients who did and did not develop recurrence are summarized in Table 5. Patients with recurrence had significantly higher pathologic lymph node status $(\mathrm{P}=0.03)$, pathologic stage $(\mathrm{P}=0.02)$, mean number of positive lymph nodes $(\mathrm{P}=0.01)$, and mean percentage of lymph nodes positive $(\mathrm{P}=0.02)$.
Interestingly, two patients had preoperative histopathologic diagnosis of squamous cell carcinoma with one considered Siewert type I clinically. Both were surgically approached transhiatally with laparotomy and cervical incisions and had total esophagectomy with proximal gastrectomy. Gastric pull-up with cervical anastomosis was done for both patients. At 1 year, both patients were alive with no evidence of disease. However, one patient developed nodal and liver recurrence 501 days post-operatively.

\section{DISCUSSION}

The majority of the patients in this study had upper gastrointestinal obstruction. Based on body mass index (BMI) and albumin they were generally not nutritionally replete. However, detailed preoperative nutritional assessment tools should be used to better define and manage their nutritional risks: large-scale nutritional screening of cancer outpatients has shown that patients with esophagogastric cancer had very high nutritional risk..$^{25}$ 
Chronic obstructive pulmonary disease (COPD) affects surgical management of EGJ adenocarcinoma as it increases risks of pulmonary complications, particularly following transthoracic approaches. ${ }^{26}$ Low predicted forced expiratory volume at 1 second (FEV1 \% predicted) has been associated with increased pulmonary morbidity following esophagectomy. ${ }^{26,27}$ Preoperative pulmonary function testing (PFT) is a useful aid in selecting surgical approach. In this study, although $11 \%$ had COPD and the majority being smokers with an average of 33 pack-years, most patients had normal PFT.

The predominance of Siewert types II and III tumors in this study was consistent with reports from other countries that have reported more Siewert type I tumors in Western populations, and types II and III among Asians., ${ }^{1,2,6,28-30}$ This may account for the limited number of patients with history of GERD and associated intestinal metaplasia or Barrett's esophagus in the study. Type I tumors are considered as distal esophageal adenocarcinoma arising from Barrett's esophagus, which occurs in patients with longstanding GERD. ${ }^{1,2}$

Correlation between clinical and pathologic staging in the study was poor. This can be attributed to use of endoscopy and computed tomography alone in the preoperative staging. Assessment of depth of mural involvement and lymph node metastases is limited with computed

Table 5. Clinicopathologic and treatment factors in patients with or without recurrence after curative resection for esophagogastric junction adenocarcinoma

\begin{tabular}{|c|c|c|c|}
\hline Variable & $\begin{array}{l}\text { With recurrence }(n=21) \\
\%(n / N)\end{array}$ & $\begin{array}{l}\text { Without recurrence }(\mathrm{n}=15) \\
\%(\mathrm{n} / \mathrm{N})\end{array}$ & $P$ value \\
\hline Age (mean, years) & $56.5(n=20)$ & $50.1(n=15)$ & $0.23^{*}$ \\
\hline $\mathrm{T}$ & & & $0.33^{\ddagger}$ \\
\hline T1 & 0 & 0 & \\
\hline $\mathrm{T} 2$ & $4.8(1 / 21)$ & $13.3(2 / 15)$ & \\
\hline T3 & $85.7(18 / 21)$ & $86.7(13 / 15)$ & \\
\hline $\mathrm{T} 4 \mathrm{a}$ & $9.5(2 / 21)$ & 0 & \\
\hline $\mathrm{T} 4 \mathrm{~b}$ & 0 & 0 & \\
\hline $\mathrm{N}$ & & & $0.03^{\ddagger}$ \\
\hline NO & $14.3(3 / 21)$ & $26.7(4 / 15)$ & \\
\hline N1 & $4.8(1 / 21)$ & $40(6 / 15)$ & \\
\hline N2 & $38.1(8 / 21)$ & $20(3 / 15)$ & \\
\hline N3 & $38.1(8 / 21)$ & $13.3(2 / 15)$ & \\
\hline Unknown & $4.8(1 / 21)$ & 0 & \\
\hline Pathologic stage & & & $0.02^{\ddagger}$ \\
\hline IA & 0 & 0 & \\
\hline IB & $4.8(1 / 21)$ & $6.7(1 / 15)$ & \\
\hline$\| \mathrm{A}$ & 0 & 0 & \\
\hline IIB & $9.5(2 / 21)$ & $20(3 / 15)$ & \\
\hline IIIA & $4.8(1 / 21)$ & $46.7(7 / 15)$ & \\
\hline IIIB & $33.3(7 / 21)$ & $13.3(2 / 15)$ & \\
\hline IIIC & $42.9(9 / 21)$ & $13.3(2 / 15)$ & \\
\hline Unknown & $4.8(1 / 21)$ & 0 & \\
\hline No. of lymph nodes positive (mean) & $7.6(n=20)$ & $2.3(n=15)$ & $0.01^{*}$ \\
\hline Percentage of lymph nodes positive (mean) & $47.5(n=20)$ & $20.9(n=15)$ & $0.02 *$ \\
\hline Proximal margin status & & & $1.00^{+}$ \\
\hline Positive & $4.8(1 / 21)$ & $6.7(1 / 15)$ & \\
\hline Negative & $95.2(20 / 21)$ & $93.3(14 / 15)$ & \\
\hline Margin length (mean, $\mathrm{cm}$ ) & $2.7(n=11)$ & $3(n=11)$ & $0.82^{*}$ \\
\hline Distal margin status & & & $0.50^{+}$ \\
\hline Positive & $9.5(2 / 21)$ & 0 & \\
\hline Negative & $90.5(19 / 21)$ & $100(15 / 15)$ & \\
\hline Margin length (mean, cm) & $3.4(n=5)$ & $8.2(n=8)$ & $0.11^{*}$ \\
\hline Circumferential/radial margin & & & $0.32^{\ddagger}$ \\
\hline Positive & $9.5(2 / 21)$ & $6.7(1 / 15)$ & \\
\hline Negative & $4.8(1 / 21)$ & $13.3(2 / 15)$ & \\
\hline Close & 0 & $13.3(2 / 15)$ & \\
\hline Unknown & $85.7(18 / 21)$ & $66.7(10 / 15)$ & \\
\hline Margin width (mean, $\mathrm{cm}$ ) & $0(n=2)$ & $0.06(n=4)$ & \\
\hline Pathologic Siewert type & & & $0.30^{\ddagger}$ \\
\hline Type I & $4.8(1 / 21)$ & $6.7(1 / 15)$ & \\
\hline Type II & $42.9(9 / 21)$ & $66.7(10 / 15)$ & \\
\hline Type III & $52.4(11 / 21)$ & $26.7(4 / 15)$ & \\
\hline
\end{tabular}


Table 5. Clinicopathologic and treatment factors in patients with or without recurrence after curative resection for esophagogastric junction adenocarcinoma (continued)

\begin{tabular}{|c|c|c|c|}
\hline Variable & $\begin{array}{c}\text { With recurrence }(n=21) \\
\%(n / N)\end{array}$ & $\begin{array}{l}\text { Without recurrence }(n=15) \\
\%(n / N)\end{array}$ & $P$ value \\
\hline $\begin{array}{l}\text { Lymphovascular space invasion } \\
\text { Positive } \\
\text { Negative } \\
\text { Unknown }\end{array}$ & $\begin{array}{c}19(4 / 21) \\
0 \\
80.9(17 / 21) \\
\end{array}$ & $\begin{array}{c}0 \\
0 \\
100(15 / 15) \\
\end{array}$ & $1.00^{\dagger}$ \\
\hline $\begin{array}{l}\text { Histologic grade } \\
\text { G1 } \\
\text { G2 } \\
\text { G3 } \\
\text { Unknown }\end{array}$ & $\begin{array}{c}38.1(8 / 21) \\
28.6(6 / 21) \\
28.6(6 / 21) \\
4.8(1 / 21) \\
\end{array}$ & $\begin{array}{c}26.7(4 / 15) \\
33.3(5 / 15) \\
33.3(5 / 15) \\
6.7(1 / 15) \\
\end{array}$ & $0.79 \ddagger$ \\
\hline $\begin{array}{l}\text { Surgical Approach } \\
\text { Thoracoabdominal } \\
\text { Transhiatal } \\
\text { Laparotomy + Cervical } \\
\text { Unknown }\end{array}$ & $\begin{array}{c}52.4(11 / 21) \\
9.5(2 / 21) \\
19(4 / 21) \\
19(4 / 21) \\
\end{array}$ & $\begin{array}{c}86.7(13 / 15) \\
6.7(1 / 15) \\
6.7(1 / 15) \\
0 \\
\end{array}$ & $0.34^{\ddagger}$ \\
\hline $\begin{array}{l}\text { Surgical Resection } \\
\text { Distal esophagectomy + total gastrectomy } \\
\text { Total thoracic esophagectomy + proximal gastrectomy } \\
\text { Distal esophagectomy + proximal gastrectomy }\end{array}$ & $\begin{array}{l}71.4(15 / 21) \\
19(4 / 21) \\
9.5(2 / 21) \\
\end{array}$ & $\begin{array}{c}73.3(11 / 15) \\
20(3 / 15) \\
6.7(1 / 15) \\
\end{array}$ & $0.95^{\ddagger}$ \\
\hline En bloc resection of other organs & $42.9(9 / 21)$ & $26.7(4 / 15)$ & $0.48^{\dagger}$ \\
\hline Adjuvant chemo/chemoradiotherapy & $50(9 / 18)$ & $54.5(6 / 11)$ & $0.63^{\ddagger}$ \\
\hline $\begin{array}{l}\text { Surgeon type } \\
\text { Trainee } \\
\text { Consultant }\end{array}$ & $\begin{array}{c}23.8(5 / 21) \\
76.2(16 / 21)\end{array}$ & $\begin{array}{c}26.7(4 / 15) \\
73.3(11 / 15)\end{array}$ & $1.00^{\dagger}$ \\
\hline
\end{tabular}

*One way analysis of variance (ANOVA)

'Fisher's exact test

${ }^{\ddagger}$ Chi-squared test

Note: Bold font, statistically significant $P$ value

tomography compared to endoscopic ultrasonography, ${ }^{31}$ which was infrequently used. Computed tomography, however, remains important in defining surgical resectability. ${ }^{32}$

Staging laparoscopy has high sensitivity for resectability and avoids unnecessary laparotomy in up to $20 \%$ of cases. ${ }^{33}$ This study suggests that staging laparoscopy is underutilized: a majority of the tumors were locally advanced and had inaccurate clinical staging. This apparent underutilization may be because laparoscopy was not commonly used in the institution in the earlier years of the 10-year inclusion period. Another possible reason is that the majority of patients had upper gut obstruction that necessitated an operative enteral access, regardless of tumor resectability. In the left thoracoabdominal approach, initial small laparotomy to assess tumor resectability substituted for staging laparoscopy.

With most of the tumors in this series being Siewert types II and III, the observed preference for left thoracoabdominal approach and total gastrectomy with distal gastrectomy is understandable. Siewert types II and III tumors are generally regarded as gastric malignancies, for which extended gastrectomy with resection of distal esophagus is advocated. ${ }^{1,2,10}$ For select Siewert type II tumors, proximal gastrectomy with distal esophageal resection is an option. ${ }^{2}$ Extended total or proximal gastrectomy can be approached via laparotomy with transhiatal resection of distal esophagus or via a left thoracoabdominal incision. ${ }^{1,2,12}$ The observed preference for total thoracic esophagectomy with proximal gastrectomy via non-transthoracic approach in Siewert type I tumors is consistent with the view that they are primarily esophageal malignancies. ${ }^{1,2}$

The lack of an observed difference in histopathologic and clinical findings between surgical approaches and resections is generally in line with studies from other countries. $^{7-10,13} \mathrm{~A}$ meta-analysis on transthoracic versus non-transthoracic resection for EGJ cancer showed no significant differences in survival, postoperative morbidity and mortality. ${ }^{13}$ Although increased morbidities have been associated with thoracoabdominal approaches in previous studies, this was not the case in this study. ${ }^{12}$

A significant difference was observed in distal margin status and length in favor of transthoracic approaches, but this is likely due to the nature of the associated surgical resection and may not be of clinical importance. With the surgical approach, the critical issue is the need for a thoracotomy for adequate access to the proximal extent of the tumor in the chest. An adequate distal margin should be equally achievable with transthoracic and non-transthoracic approaches. As might be expected, patients who underwent distal compared to total thoracic esophagectomy had shorter proximal margin length. This highlights the need for accurate intraoperative assessment of proximal tumor extent 
and liberal use of frozen section biopsy to ensure adequate margins. Patient selection is important and less than total gastrectomy may be best applied only in those with early disease. ${ }^{2}$ Data on type and extent of lymph node dissection was not included in operative and histopathologic reports and could not be included in this study. This information is important because nodal involvement and its clearance predict survival. ${ }^{2,8,21-23,30,34}$ Given the unique location of EGJ adenocarcinoma, the pattern of lymph node metastases has been shown to vary according to Siewert type..$^{35}$ En bloc lymphadenectomy for EGJ adenocarcinoma frequently included removal of the lower posterior mediastinal nodes along with standard D2 dissection (lymph node stations $1-12$ of the Japanese classification). ${ }^{2}$

Despite the majority of patients in this series having locally advanced disease, only one patient received neoadjuvant chemotherapy. This treatment can downstage tumors, facilitate curative resection, and improve survival. ${ }^{14-16,20} \mathrm{~A}$ meta-analysis that included 14 RCTs has demonstrated improved survival with preoperative chemotherapy compared to surgery alone [HR $0.81,95 \%$ confidence interval 0.73 0.89, $\mathrm{p}<0.001)$ in EGJ adenocarcinoma patients. ${ }^{20}$ Larger survival advantages were seen with chemoradiotherapy. ${ }^{20}$ In the CROSS trial, neoadjuvant chemoradiotherapy using carboplatin and paclitaxel was associated with improved overall survival compared to surgery alone in patients with esophageal or esophagogastric junction adenocarcinoma (HR 0.73, CI 0.55-0.98). ${ }^{36}$ Given this evidence, the advanced stage of EGJ adenocarcinoma in this institution and its associated poor survival, we should consider the use of preoperative therapy in appropriately selected patients. The predominant use of outright surgery in EGJ adenocarcinoma in this series does not parallel the current National Comprehensive Cancer Network esophageal/EGJ cancer guidelines that have preoperative chemoradiotherapy as the preferred approach. ${ }^{3}$

Among patients with available data, majority received adjuvant chemotherapy that was largely cisplatin and fluorouracil-based. This is consistent with most studies on adjuvant therapy for EGJ adenocarcinoma that used cisplatin and/or fluorouracil or its derivatives. ${ }^{17-19}$ Previous studies observed improved survival for adjuvant chemotherapy or chemoradiotherapy. ${ }^{17-19}$ The lack of a significant difference in recurrence observed in this study between those who did or did not receive adjuvant therapy may be due to the small number of patients with such data, limited follow-up, and variable and often incomplete chemotherapy/chemoradiotherapy regimens. No comparison could be made for overall survival due to the paucity of patients with long-term follow-up.

Around $60 \%$ of patients with follow-up data in this study experienced a recurrence; consistent with reports that EGJ adenocarcinomas are prone to recur despite curative resections. ${ }^{37}$ Mean time to recurrence in this series was in line with literature, with most recurrences occurring within a year of surgery. ${ }^{37}$ In contrast with reports showing hematogenous recurrences being most common, ${ }^{37}$ locoregional and, in particular, peritoneal recurrences were most common in this study. This may be due to differences in predominant Siewert types and surveillance protocols. This is in line with a previous study with mostly Siewert type III tumors, which observed predominantly peritoneal recurrences. ${ }^{21}$

The factors that were significantly different between those who did and did not develop recurrence - namely, pathologic nodal status, number and percentage of positive lymph nodes and stage - all relate to pathologic nodal burden; and are in agreement with reports showing worse prognosis with increased nodal disease. ., $7,8,21-23,30,34^{A}$ previous study suggested that cure was limited to patients with less than 6 involved lymph nodes. ${ }^{21}$ In Chinese patients with EGJ adenocarcinoma, $\geq 16$ positive lymph nodes, lymph node ratio $>0.2$, and overall pathologic stage were poor survival predictors. ${ }^{23}$ In this study, more patients who experienced a recurrence were pathologic N3, pathologic stage IIIB/IIIC, and had lymph node ratio close to 0.5 . R0 resection status (no macroscopic and microscopic evidence of residual tumor) is a frequently reported predictor of survival outcomes. ${ }^{2,8,21,22,34}$ However, due to infrequent documentation of completeness of gross resection and circumferential or radial resection margins, it could not be considered in this study.

Differences in in-hospital mortality between different resection types should be interpreted with caution due to the small numbers for some surgeries. In this study, longterm survival outcomes were not available. Estimated 5-year overall survival for the disease is approximately $20 \% .{ }^{1}$ Oneyear overall survival among those with available data ranged from $78-87 \%^{22,23}$ as reported in literature and reflected the overall poor prognosis of the disease. The significantly different 1-year overall survival across Siewert types with worse outcomes with type I in this study is consistent with a Korean report showing worse prognosis for type I tumors. ${ }^{28}$ However, this must be interpreted with caution due to the small number of type I tumors in this series and poor followup. Most reports show no difference in survival outcomes according to Siewert type. $8,21,22,30,37$

The incomplete and inconsistent documentation of clinicopathologic data in many of the patients included in the study is evident. Standardized patient data forms and histopathologic reports must be used. Patient follow-up needs improvement to more accurately assess treatment outcomes. This may be facilitated by patient navigation.

This institution's surgical approach and extent of resection for EGJ adenocarcinoma have not changed significantly in recent years, since the disease and patient characteristics have remained largely the same. Majority is still managed with distal esophagectomy and total gastrectomy via left thoracoabdominal approach. However, there has been increasing use of the transhiatal approach with a laparotomy incision and with esophagojejunal or esophagogastric anastomosis in the lower mediastinum due to availability of stapling device for anastomosis. Both the current National 
Comprehensive Cancer Network (NCCN) and Japanese Esophageal Society and Japanese Gastric Cancer Association guidelines have no strict and standard recommendation on approach and resection extent in EGJ cancer as choice largely depends on several factors including tumor location and surgeon experience. ${ }^{3,38-39}$ In recent years, however, there is a trend towards increased application of neoadjuvant therapy for cases managed in the institution, either as preoperative chemoradiotherapy or perioperative chemotherapy, consistent with recent NCCN recommendations. ${ }^{3}$ Use of preoperative chemoradiotherapy is increasing in particular, following the results of the CROSS trial showing improved median survival of about 16 months over surgery alone approach. ${ }^{36}$

\section{CONCLUSIONS}

EGJ adenocarcinomas in the Philippine General Hospital are frequently locally advanced. Since outcomes are comparable across various surgical approaches and resections, the choice of surgery should depend on tumor characteristics particularly Siewert type, patient comorbidities and functional status. Surgery remains central in management. However, in light of recent evidence and the generally poor prognosis of the disease, multimodality approach should be applied. Improved documentation of clinical data will facilitate assessment of patient and institutional performance.

\section{Statement of Authorship}

All authors participated in data collection and analysis, and approved the final version submitted.

\section{Author Disclosure}

All authors declared no conflicts of interest.

\section{Funding Source}

No external funding for this paper.

\section{REFERENCES}

1. Fox MP, van Berkel V. Management of gastroesophageal junction tumors. Surg Clin North Am. 2012 Oct; 92(5):1199-212. doi: 10.1016/j. suc.2012.07.011.

2. Siewert JR, Feith M, Werner M, Stein HJ. Adenocarcinoma of the esophagogastric junction: Results of surgical therapy based on anatomical/topographic classification in 1,002 consecutive patients. Ann Surg. 2000 Sep; 232(3):353-61. doi: 10.1097/00000658-20000900000007.

3. National Comprehensive Cancer Network. NCCN Clinical Practice Guidelines in Oncology. Esophageal and Esophagogastric Junction Cancers [Internet]. [cited 2020 Mar 15]. Available from: https://www. nccn.org/professionals/physician_gls/pdf/esophageal.pdf.

4. Vial M, Grande L, Pera M. Epidemiology of adenocarcinoma of the esophagus, gastric cardia, and upper gastric third. Recent Results Cancer Res. 2010; 182:1-17. doi: 10.1007/978-3-540-705796 1.

5. Deans C, Yeo MSW, Soe MY, Shabbir A, Ti TK, So JBY. Cancer of the gastric cardia is rising in incidence in an Asian population and is associated with adverse outcome. World J Surg. 2011 Mar; 35(3): 617-24. doi: 10.1007/s00268-010-0935-0.
6. Kusano C, Gotoda T, Khor CJ, Katai H, Kato H, Taniguchi H, et al. Changing trends in the proportion of adenocarcinoma of the esophagogastric junction in a large tertiary referral center in Japan. J Gastroenterol Hepatol. 2008 Nov; 23(11):1662-5.

7. Barbour AP, Rizk NP, Gonen MG, Tang L, Bains MS, Rusch VW, et al. Adenocarcinoma of the gastroesophageal junction: Influence of esophageal resection margin and operative approach on outcome. Ann Surg. 2007 Jul; 246(1):1-8. doi: 10.1097/01.sla.0000255563.65157.d2.

8. Birla R, Iosif C, Mocanu A, Gindea C, Hoară P, Panaitescu E, et al. Long-term survival after eso-gastrectomy for esophagogastric junction adenocarcinoma: Prospective study. Chirurgia (Bucur). 2008 Nov-Dec; 103(6):635-42.

9. Johansson J, Djerf P, Oberg S, Zilling T, von Holstein CS, Johnsson $\mathrm{F}$, et al. Two different surgical approaches in the treatment of adenocarcinoma at the gastroesophageal junction. World J Surg. 2008 Jun; 32(6):1013-20. doi: 10.1007/s00268-008-9470-7.

10. Nakamura T, Ide H, Eguchi R, Ota M, Shimizu S, Isono K. Adenocarcinoma of the esophagogastric junction: A summary of responses to a questionnaire on adenocarcinoma of the esophagus and the esophagogastric junction in Japan. Dis Esophagus. 2002; 15(3): 219-25. doi: 10.1046/j.1442-2050.2002.00262.x.

11. Suttie SA, Li AG, Quinn M, Park KG. The impact of operative approach on outcome of surgery for gastro-oesophageal tumours. World J Surg Oncol. 2007; Aug; 5:95. doi: 10.1186/1477-7819-5-95.

12. Sasako M, Sano T, Yamamoto S, Sairenji M, Arai K, Kinoshita T, et al. Left thoracoabdominal approach versus abdominal-transhiatal approach for gastric cancer of the cardia or subcardia: A randomized controlled trial. Lancet Oncol. 2006 Aug; 7(8):644-51. doi: 10.1016/ S1470-2045(06)70766-5.

13. Yang K, Chen HN, Chen XZ, Lu QC, Pan L, Liu J, et al. Transthoracic resection versus non-transthoracic resection for gastroesophageal junction cancer: A meta-analysis. Plos One. 2012; 7(6):e37698. doi: 10.1371/journal.pone.0037698.

14. Cunningham D, Allum WH, Stenning SP, Thompson JN, Van de Velde $\mathrm{CJH}$, Nicolson M, et al. Perioperative chemotherapy versus surgery alone for resectable gastroesophageal cancer. N Engl J Med. 2006 Jul; 355(1):11-20. doi: 10.1056/NEJMoa055531.

15. Ychou M, Boige V, Pignon JP, Thompson JN, Conroy T, Bouché $\mathrm{O}$, Lebreton G, et al. Perioperative chemotherapy compared with surgery alone for resectable gastroesophageal adenocarcinoma: An FNCLCC and FFCD multicenter phase III trial. J Clin Oncol. 2011 May; 29(13):1715-21. doi: 10.1200/JCO.2010.33.0597.

16. van Hagen P, Hulshof MCCM, van Lanschot JJB, Steyerberg EW, van Berge Henegouwen MI, Wijnhoven BPL, et al. Preoperative chemoradiotherapy for esophageal or junctional cancer. N Engl J Med. 2012 May; 366(22):2074-84. doi: 10.1056/NEJMoa1112088.

17. Smalley SR, Benedetti JK, Haller DG, Hundahl SA, Estes NC, Ajani JA, et al. Updated analysis of SWOG-directed intergroup study 0116 : A phase III trial of adjuvant radiochemotherapy versus observation after curative gastric cancer resection. J Clin Oncol. 2012 Jul; 30(19): 2327-33. doi: 10.1200/JCO.2011.36.7136.

18. Sakuramoto S, Sasako M, Yamaguchi T, Kinoshita T, Fujii M, Nashimoto A, et al. Adjuvant chemotherapy for gastric cancer with S-1, an oral fluoropyrimidine. N Engl J Med. 2007 Nov; 357(18):1810-20. doi: 10.1056/NEJMoa072252.

19. Bang YJ, Kim YW, Yang HK, Chung HC, Park YK, Lee KH, et al. Adjuvant capecitabine and oxaliplatin for gastric cancer after D2 gastrectomy (CLASSIC): a phase 3 open-label, randomized controlled trial. Lancet. 2012 Jan; 379(9813):315-21. doi: 10.1016/ S0140-6736(11)61873-4.

20. Ronellenfitsch U, Schwarzbach M, Hofheinz R, Kienle P, Kieser M, Slanger TE, et al. Preoperative chemo(radio)therapy versus primary surgery for gastroesophageal adenocarcinoma: systematic review with meta-analysis combining individual patient and aggregate data. Eur J Cancer. 2013 Oct; 49(15):3149-58. doi: 10.1016/j.ejca.2013.05.029.

21. Pedrazzani C, Pasini F, Giacopuzzi S, Bernini M, Ruzzenente A, Festini M, et al. Surgical treatment of gastro-esophageal junction adenocarcinoma: long-term results of a single Italian center. G Chir. 2004 Oct; 25(10):325-33. 
22. de Manzoni G, Pedrazzani C, Pasini F, Di Leo A, Durante E, Castaldini $\mathrm{G}$, et al. Results of surgical treatment of adenocarcinoma of the gastric cardia. Ann Thorac Surg. 2002 Apr; 73(4):1035-40. doi: 10.1016/s0003-4975(01)03571-8.

23. Zhang YF, Shi J, Yu HP, Feng AN, Fan XS, Lauwers GY, et al. Factors predicting survival in patients with proximal gastric carcinoma involving the esophagus. World J Gastroenterol. 2012 Jul; 18(27): 3602-9. doi: 10.3748/wjg.v18.i27.3602.

24. Laudico AV, Mirasol-Lumague M, Medina V, Mapua CA, Valenzuela FG, Pukkala E. 2015 Philippine Cancer Facts and Figures. Manila: Philippine Cancer Society [Internet]. [cited 2020 Apr 18]. Available from: http://www.philcancer.org.ph/wp-content/ uploads/2017/07/2015-PCS-Ca-Facts-Estimates_CAN090516.pdf.

25. Bozzetti F, Mariani L, Lo Vullo S; SCRINIO Working Group, Amerio ML, Biffi R, Caccialanza G, et al. The nutritional risk in oncology: A study of 1,453 cancer outpatients. Support Care Cancer. 2012 Aug; 20(8):1919-28. doi: 10.1007/s00520-012-1387-x.

26. Jiao WJ, Wang TY, Gong M, Pan H, Liu YB, Liu ZH. Pulmonary complications in patients with chronic obstructive pulmonary disease following transthoracic esophagectomy. World J Gastroenterol. 2006 Apr; 12(16):2505-9. doi: 10.3748/wjg.v12.i16.2505.

27. Ferguson MK, Durkin AE. Preoperative prediction of the risk of pulmonary complications after esophagectomy for cancer. J Thorac Cardiovasc Surg. 2002 Apr; 123(4):661-9.

28. Chung JW, Lee GH, Choi KS, Kim D-H, Jung KW, Song HJ, et al. Unchanging trend of esophagogastric junction adenocarcinoma in Korea: experience at a single institution based on Siewert's classification. Dis Esophagus. 2009; 22(8):676-81. doi: 10.1111/j.14422050.2009.00946.x.

29. Hasegawa S, Yoshikawa T, Cho H, Tsuburaya A, Kobayashi O. Is adenocarcinoma of the esophagogastric junction different between Japan and Western countries? The incidence and clinicopathologic features at a Japanese high-volume cancer center. World J Surg. 2009 Jan; 33(1):95-103. doi: 10.1007/s00268-008-9740-4

30. Hosokawa Y, Kinoshita T, Konishi M, Takahashi S, Gotohda N, Kato Y, et al. Clinicopathological features and prognostic factors of adenocarcinoma of the esophagogastric junction according to Siewert classification experiences at a single institution in Japan. Ann Surg Oncol. 2012 Feb; 19(2):677-83. doi: 10.1245/s10434-011-1983-x.
31. Holden A, Mendelson R, Edmunds S. Pre-operative staging of gastroesophageal junction carcinoma:comparison of endoscopic ultrasound and computed tomography. Australas Radio. 1996 Aug; 40(3):206-12. doi: 10.1111/j.1440-1673.1996.tb00386.x.

32. Terrier F, Schapira C, Fuchs WA. CT assessment of operability in carcinoma of the oesophagogastric junction. Eur J Radiol. 1984 May; $4(2): 114-7$.

33. de Graaf GW, Ayantunde AA, Parsons SL, Duffy JP, Welch NT. The role of staging laparoscopy in oesophagogastric cancers. Eur J Surg Oncol. 2007 Oct; 33(8):988-92. doi: 10.1016/j.ejso.2007.01.007.

34. Bosing NM, Heise JW, Goretzki PE, Sarbia M, Röher H-D. Adenocarcinoma of the esophagogastric junction: Prognostic factors and results of primary surgery. Chirurg. 2004 Nov; 75(11):1088-97. doi: 10.1007/s00104-004-0840-x.

35. Dresner SM, Lamb PJ, Bennett MK, Hayes N, Griffin SM. The pattern of metastatic lymph node dissemination from adenocarcinoma of the esophagogastric junction. Surgery. 2001 Jan; 129(1):103-9. doi: $10.1067 / \mathrm{msy} .2001 .110024$.

36. Shapiro J, van Lanschot JBB, Hulshof MCCM, van Hagen P, van Berge Henegouwen MI, Wijnhoven BPL, et al. Neoadjuvant chemoradiotherapy plus surgery versus surgery alone for oesophageal or junctional cancer (CROSS): long-term results of a randomised controlled trial. Lancet Oncol. 2015 Sep; 16(9):1090-8. doi: 10.1016/ S1470-2045(15)00040-6.

37. Wayman J, Bennett MK, Raimes SA, Griffin SM. The pattern of recurrence of adenocarcinoma of the oesophago-gastric junction. $\mathrm{Br}$ J Cancer. 2002 Apr; 86(8):1223-9. doi: 10.1038/sj.bjc.6600252.

38. Kitagawa Y, Uno T, Oyama T, Kato K, Kato H, Kawakubo H, et al. Esophageal cancer practice guidelines 2017 edited by the Japan Esophageal Society: Part 2. Esophagus. 2019 Jan; 16(1):25-43. doi: 10.1007/s10388-018-0642-8.

39. Japanese Gastric Cancer Association. Japanese gastric cancer treatment guidelines 2018 (5th edition). Gastric Cancer. 2020 Feb. doi: 10.1007/s10120-020-01042-y. 